
\title{
$\angle S$ Research Square \\ B2M gene knockout in HEK293T cells by non-viral delivery of CRISPR-Cas9 for the induction of universal cell
}

\section{Maryam Ranjbar}

Shiraz Medical School: Shiraz University of Medical Sciences

Farshid Amiri

Shiraz Medical School: Shiraz University of Medical Sciences

Marjan Nourigorji

Shiraz Medical School: Shiraz University of Medical Sciences

Farid Torabizadeh

Shiraz Medical School: Shiraz University of Medical Sciences

Mahintaj Dara

Shiraz Medical School: Shiraz University of Medical Sciences

Mehdi Dianatpour ( $\triangle$ maryamranjbar845@gmail.com )

Shiraz Medical School: Shiraz University of Medical Sciences

\section{Research Article}

Keywords: Stem cell therapy, Graft rejection, MHC class I, B2M, CRISPR/Cas9

Posted Date: June 7th, 2021

DOI: https://doi.org/10.21203/rs.3.rs-595457/v1

License: (1) This work is licensed under a Creative Commons Attribution 4.0 International License. Read Full License 


\section{Abstract}

Allogeneic stem cells have been introduced as a potential approach to generate grafts in regenerative medicine. But the clinical usage of them is limited due to the risk of immune rejection that is induced by the incompatibility of human leukocyte antigens (HLAs) between donors and recipients. To overcome this limitation, we knocked out the $\beta 2$ microglobulin (B2M) gene which is crucial for HLA class I expression, using CRISPR/Cas9 approach. Non-viral transfer of two gRNAs targeting exon 1 and intron 1 in B2M gene caused in large and predictable deletion in region between two loci, which was defined by sequencing and polymerase chain reaction (PCR). Furthermore, results revealed that roughly $11.11 \%$ and $22.22 \%$ of the GFP expressing cells reflecting a homozygous and heterozygous pattern of genomic modifications. We demonstrated that the dual guide RNA approach is a simple and efficient method for gene disruption. Significantly, these engineered hypoimmunogenic cells could be proposed as universal cells that are not distinguishable to the recipient immune system in cell therapy and transplantation.

\section{Introduction}

Organ transplantation (OTP) is the primary, yet a high-risk medical procedure to treat patients with intractable diseases and organ failures [1]. However, the OTP still faces major restrictions including the scarcity of immunologically suitable donors, which has drastically confided its application. In order to address the issues associated with the OTP, autologous and allogenic stem cells were envisioned to be alternative and effective approaches [2,3]. In principle, allogeneic cell products is thought to be the more advantageous solution compared with autologous cell products by providing safety and consistency products [4]. But the transplant rejection caused by human leukocyte antigen (HLA) mismatching between donor and recipient is a main challenge. Primarily, host T cells could activate immune responses against donor major/minor histocompatibility antigens, leading to rapid rejection. Furthermore, the possibility of graft versus host disease (GVHD) development should be considered [5].

Previous studies have demonstrated that precluding mismatch HLA by downregulated or complete abrogation of donor HLA class I expression would reduce graft rejection [6, 7]. Disrupting the expression of HLA type I by targeting the $\beta 2$-microglobulin (B2M) locus, which encoding invariable and pivotal component of HLA I, would result in the incomplete formation of defective and thus led to address immune rejection. Thus far, several attempts utilizing various methods including the Cre-LoxP system [8], small interfering RNA (siRNA) [9], transcription activator-like effector nucleases (TALEN) [10, 11], and zinc finger nuclease (ZFN) [12], have exhibited successful results at disrupting the expression of HLA class I and as a result its display on the surface of the cell. Despite much development, these approaches were encountered with limitations such as unintended recombination via the Cre-LoxP system [8, 13], and incomplete deletion or low efficacy in knocking out target genes using the RNAi method [14]. Furthermore, the arduous task of designing the TALE and ZFN nucleases along with the aforementioned shortcoming has rendered a lessen usage of those methods for either research or therapeutic purposes. To address the limitations of previous technologies, clustered regularly interspaced short palindromic repeats (CRISPR) system has been introduced as a relatively new technology. Due to the simplicity and programmability of 
the system has developed into a versatile genome-editing tool and has demonstrated promising results. The CRISPR system is constituted of ribonucleoprotein complex, which cleaves double-stranded DNA molecules harboring sequences complementary to the guide RNA (crRNA) within the ribonuclease. So, in CRISPR/Cas9 system, the DNA recognition is only dependent on the presence of an engineered 20nucleotide sequence, leading to vast application in therapeutic trials. An easy way in utilizing CRISPR/Cas 9 technology is designing two gRNAs to simultaneously target two loci in desired gene and consequently creating complete and large deletion. CRISPR/Cas9 system with single or dual gRNAs approach could generate double strand breaks (DSBs) and trigger non-homologous end joining (NHEJ) of repair pathway. This error-prone pathway could induce indel mutations and consequently result in gene knockout $[6,15]$.

In this study, the honing capability of the CRISPR/Cas9 system was exploited to target the B2M gene of the HEK293T cell line via two gRNAs, and further demonstrate an efficient knocked out gene, signifying its capability to directly cleave any targeted loci. As a first step, we designed and cloned two separate gRNAs into two separate PX458 vector with green fluorescent protein (GFP) marker. It was predicted that the combination of two gRNAs that would introduce DSBs in region $\sim 2.2 \mathrm{~kb}$ between the two loci. After co transfection of vectors into HEK293T cells, the straightforward PCR method was employed to determine the zygosity status of the cells and the result further confirmed by sequencing (Fig 1). It appears homozygous B2M (B2M-/-) null stem cells could employ as a source of universal and immunocompatible cells to use in cell therapy for the treatment of variety of diseases without considering of recipient HLA.

\section{Materials And Methods}

\subsection{Strains and reagents:}

The T4 DNA ligase (400000 U/mL, NEB \#M0202S), and the Bbsl restriction enzyme (10000 U/mL, NEB \# R0539S) was purchased from the New England Biolabs (NEB) company to carry out the ligation and digestions respectively. The pSpCas9 (BB)-2A-GFP (PX458) (Plasmid \# 48138) was supplied by Prof. A. Mani (Yale Cardiovascular Research Center, University of Yale, New Haven, Connecticut, USA). Human embryonic stem cell (HEK) 293T was provided from the biotechnology research center in Shiraz University of Medical Sciences, Iran. Lipofectamine 2000 (Invitrogen \# 11668027) and Opti-MEM (Gibco \# 31985) was purchased from Thermofisher Company to meet the requirement of an efficient transfection process.

\subsection{Methods}

\section{Designing guide RNAs and constructing the gRNAs expressing vector}

The online CRISPR tool of http://crispor.tefor.net/ was used to design a pair of guide RNAs targeting separate sites within B2M exon 1 (BME) (Table 1). The two gRNAs were designed to target distinct sites separated approximately $2.2 \mathrm{~kb}$ within the B2M gene located in chromosome 15 . The gRNA_ 1 and 
gRNA_2 harbored complementary sequences to the sense strand of exon 1 and antisense strand of intron 1 respectively. In order to facilitate the cloning process of gRNAs, an adapter sequence was incorporated according to the requirement of the vector and restriction enzyme. Furthermore, several factors were taken into account, upon designing gRNAs sequences including on-target activity, specificity, and the presence of 5'-NGG sequence at the $3^{\prime}$ end of gRNAs sequences to meet the PAM requirement of Cas9 enzyme. The double-stranded form of each guide RNA was cloned into the PX458 expression plasmid via $\mathrm{Bbsl}$ restriction sites. To monitor the transfection efficacy and the transcription of the two guide RNAs, the Green fluorescent protein (GFP), which served as a reporter protein was exploited. Digestion and ligation were performed simultanously in a total of $10 \mu \mathrm{l}$ reaction mixture per each gRNA. In order to do so, $1 \mu \mathrm{l}$ annealed gRNAs oligos, $400 \mathrm{ng}$ circular PX458 vector, $0.5 \mu \mathrm{l}$ Bbsl restriction enzyme $(10000 \mathrm{U} / \mathrm{mL}), 1 \mu \mathrm{l}$ of 10x NEB buffer, $0.5 \mu \mathrm{l}$ T4 DNA ligase (400,000 units/ml), and $1 \mu \mathrm{l}$ of 10× NEB T4 DNA ligase buffer were supplemented by $\mathrm{H} 2 \mathrm{O}$ to the total volume of $10 \mu$. Finally, the test tubes were placed in a thermal cycler with the following pre-set settings: the first step entailed 3 cycles of two-part temperature regulation, the first part was at $37^{\circ} \mathrm{C}$ for $20 \mathrm{~min}$, and the second part was at $16^{\circ} \mathrm{C}$ for $15 \mathrm{~min}$, the second step entailed one cycles of two-part temperature regulation, the first part was at $37^{\circ} \mathrm{C}$ for $10 \mathrm{~min}$, and the second part was at $55^{\circ} \mathrm{C}$ for $15 \mathrm{~min}$. Proper vector construction was confirmed by PCR using CrB2M_1 and CrB2M_2 primers (Table2). The results were further confirmed by sequencing.

\section{Cell culture and transfection}

The HEK293T cell line was selected for genetic manipulation of the B2M gene due to its high-rate transfection efficiency, docility, and fast growth. The transfection was mediated by the intrinsic properties of Lipofectamine. To do so, the cloned gRNAs were encapsulated in the Lipofectamine reagent, and the cells were simultaneously supplemented with both gRNAs. In order to investigate the transfection efficiency, the FACSAria ${ }^{\text {TM }}$ cell sorter was exploited. HEK293T cell line was cultured in Dulbecco's modified Eagle's medium (Gibco), supplemented with $10 \%$ Fetal bovine serum (Gibco) and $500 \mu$ PenicillinStreptomycin. The cell passaging was performed upon reaching $90 \%$ confluency every 2 to 3 days. Twenty-four hours prior to transfection, a six-welled plate was seeded with $5 \times 10^{5}$ cells in each well, and cultivated in $2 \mathrm{~mL}$ growth media devoid of the Penicillin-Streptomycin antibiotics. The transfections were mediated by Lipofectamine 2000 (Invitrogen) encapsulating the cloned gRNAs according to the procedure published by Pankaj K. Mandal (Mandal et al., 2014). To obtain the optimum result the final concentration of each gRNA was reduced to half.

\section{Isolation of transfected cells via fluorescence-activated cell sorting (FACS) and single cell preparation}

Forty-eight hours ensuing the transfection, the GFP expressing cells (GFP+) were separated from others via the FACS, and subsequently were cultivated in complete growth media for 3 to 4 days to expand into $80 \%$ of confluency, and then the cell suspension was subjected to serial dilution to determine the zygosity of single cells. To do so, the cell suspension with a concentration of 106 cells per $1 \mathrm{~mL}$ was provided and subsequently was serially diluted up until reaching the final concentration of 1 cell per $100 \mu \mathrm{L}$. Every single cell was supplemented with an additional $100 \mu \mathrm{L}$ growth media to propagate. 


\section{Zygosity determination via polymerase chain reaction and sequencing confirmation}

The distinct clones of cells each derived from single-cell expansion were subjected to genomic DNA extraction. The extracted DNAs were amplified by PCR using primers depicted in (Table 2). The PCR product was analyzed by gel electrophoresis and further purification. The gel-purified PCR products were cloned into a TA vector using the TOPO TA cloning kit (Thermo Fisher Scientific) according to the manufacturer's instruction for sequencing analysis. The results demonstrated the zygosity state of each clone, including homozygous, heterozygous, and wild-type states, each signifying single allele mutation, double allele mutation, and no mutation respectively.

\section{Results}

\section{The construction and verification of the guide RNAs targeting B2M gene}

gRNAs were designed to target predefined sites within sequences of B2M gene. The gRNAs were each cloned into a distinct PX458 vector with U6 polymerase III promoterusing Bbsl cleavage site, which are indicated by PX458_gRNA \#1 and PX458_ gRNA \#2. Sequencing and PCR amplification revealed correct coloning of gRNAs into vector. In this study, Cas9-gRNAs system should eliminated the expression of B2M via error prone pathway of non-homologous end joining (NHEJ).

\section{The generation of B2M null HEK293T cell line}

The $25 \%$ proportion of the cells was determined to be GFP+ whithin 48 hours of transfection (Fig 2). We used serial dilution to separate single cell clones from heterogenous cell population to assess the function of Cas9. Upon introducing Cas9-gRNAs system into cells we expect to see four potential cell lines, cell lines with no mutation, cell lines with heterozygous mutation, cell line with biallelic mutation and cell lines with homozygous mutation. Disruption of both alleles within the predicted gRNAs targeting sites resulted in the ablation of B2M gene expression.

\section{Zygosity determination B2M null HEK293T cell line}

The genomic DNA from all single cell-derived expanded clones were analyzed via PCR and sequencing. The PCR results delineated the proportion of cells with genetic alteration, signifying a segment deletion (i.e. homozygous and heterozygous states), and the proportion of cells with an intact genome, constituting the wild-type cell population. Cells with homozygote and heterozygote deletions demonstrated as single band (473bp) and double bands (473bp and 2765bp) on agarose gel electrophoresis. In addition, wild-type cells were visible as a single band (2765bp).In order to verify the PCR derived observations, the samples were sequenced and the result confirmed that approximately $11.11 \%$ and $22.22 \%$ of the GFP expressing cells contributed to the homozygous and heterozygous genome modifications respectively. The remainder proportion of the cells constituted the population with an intact genome (i.e. wild-type). Of note, due to the off-target effectiveness of the Cas 9 enzyme, a variety of small indels in several clones were further observed. 


\section{Discussion}

Here, our objective was to explore the efficiency of dual guide RNA based CRISPR/Cas9 mediated genome editing as a straightforward method for genetic manipulations, to produce HLA class 1 null donor cells that would potentially overcome the issues linked to the conventional transplantation methods. Based on the structural importance of the B2M subunit, abrogating the expression of the B2M have resulted in the improper and hence inactive formation of the HLA type $1[16,17]$. As a result of the eradication of cell surface expression of polymorphic HLA class I molecules (HLA-A,-B,-C), allograft cells have the chance to evade the host immune system and not recognized by the CD8+ T cells. The CRISPR/Cas9 system has developed to be a versatile tool for genome manipulations [18]. The system empowered us to ablate the B2M gene by simple introduction of two guide RNAs into the cell and cut out a relatively large segment of the DNA. In our study, approximately $50 \%$ of cells were targeted by the CRISPR/Cas9 constructs containing BME and BMI simultaneously, and $11-30 \%$ of targeted clones are homozygous or heterozygous. Notably, the creation of relatively large deletion within the target region would enable us to employ a fast and straightforward method such as PCR for genotyping the modified genes. Our data as well as findings from other studies clearly revealed the benefits of utilizing dual gRNAs approach in gene knockout. It was already shown that this efficient strategy can be employed to study repetitive sequences in which genetic manipulations via single guide RNA results in increased offtarget effects. In addition, the successful applicability of dual gRNA CRISPR/Cas9 technology in perturbing non-coding regions including silencers, enhancers, and long non- coding RNAs, has been reported $[6,19]$. Studies have further revealed that there is a higher probability that small indel mutations, created by the usage of a single gRNA based CRISPR/Cas system, may not lead to a loss of function in non-coding regions, resulting in an error in the investigation of such locales which constitute the majority proportions of the genetic information [6,20]. Furthermore, the utilization of single gRNAs in CRISPR/Cas9 methods leads to generation of various small insertions/deletions in targeted sequences which require arduous and inaccurate T7 endonuclease 1 (T7E1) assay to detect mismatch repair and estimate Cas 9 activity [21]. According to the previous findings, employing a single gRNA method appears to not having the ability efficient ablation of b2m in certain cell types such as $\mathrm{T}$ cells [6].

Considering the established effect of B2M knockout on complete disruption of HLA class 1, as well as its high efficiency and suitability in knockout process, we evaluated the efficiency of B2M ablation on the generation of a universal donor cells. Hong et al generated HLA class I null-cells by targeting HLA-A/B/C genes by exploiting six gRNAs simultaneously which shown to have lower efficiency in comparison with the method applied in our study. According to their results, the efficiency for co-transfection of six gRNAs into a cell, and viability of the target cells is reduced [22]. Furthermore, the subsequent selection of cells simultaneously affected by the six gRNAs will be technically difficult. In another studies conducted by Xu et al and Jang et al, the HLA-A and/or HLA-B was successfully ablated in inducible pluripotent stem cells (iPSC) instead of complete disrupting of HLA class I [23, 24]. Albeit they were able to attain less immunogenicity cells, but there will still be HLA disparity for alleles retaining between donor and recipient which should be assessed. 
Another alternative way to provide favorable source of donor cells is knocking out of the HLA class II in certain situations. Although, ablation of HLA class II by targeting CIITA gene can lead to expand immune compatibility, knocking out the HLA class II can lead to lymphopenia due to impairment of CD4+ T cells maturation $[23,25]$.

In result, the generation of universal cells using dual gRNAs approach can resolve the issues such as the shortage of suitable donors and graft rejections that are associated with other methods. Moreover, these HLA class I null-cells can serve as a source of artificial antigen-presenting cells for the production of cytotoxic T cells. However, it harbors several limitations including recognition and destruction of the cells by natural killer cells (NK cells), as HLA class I molecules serve as ligands for the NK cells. To remedy that, over expression of non-classical HLA type 1 protein has been suggested to alleviate the situation emerging from the NK cells [25]. Tumor formation arising from the HLA I deficient cells following transplantation is another dilemma to address [26]. The HLA class I deficiency may increase the risk of tumor progression and metastasis. To resolve the issue, introduction of suicide genes or artificially induced apoptosis has been suggested to prevent tumor formation. Furthermore, it has been proposed that by engineering genetically modified inducible Caspase 9 , the artificially induced apoptosis has shown more promising outcomes, as it demonstrated to be more effective, safe, and less immunogenic compared to the former approach $[8,26]$.

\section{Conclusion}

In summary, we targeted the specific sites in B2M gene via two gRNAs result in eradication of cell surface expression of polymorphic HLA class I molecules (HLA-A,-B,-C). This allograft cells have the chance to evade the host immune system and not recognized by the $C D 8+T$ cells. Previous studies have shown that these manipulated stem cells maintain their self-renewing capacity to differentiate into more specialized cells, and have demonstrated to be a promising source for replacement therapy. The process can also lead to generate a universal cell line for therapeutic aims in different patients regardless of HLA nature.

\section{Declarations}

Acknowledgment: We would like to express our very great appreciation to Dr. Mohsen Mazloom Rezaei for his valuable guidance and support and Mr. Saeed ataei for his help in writing the article. We would also to thank the all staff of the department of immunology and Student research committee in Shiraz University of Medical Sciences for their help in during the research work and financial support.

\section{Declarations:}

The authors declare that there is no conflict of interest.

\section{References}


1. Grinyó, J.M., Why is organ transplantation clinically important? Cold Spring Harbor perspectives in medicine, 2013. 3(6): p. a014985.

2. Calne, R., Challenges of organ transplantation. Transplant Proc, 2005. 37(5): p. 1979-83.

3. Heidary Rouchi, A. and Mahdavi-Mazdeh, Regenerative Medicine in Organ and Tissue Transplantation: Shortly and Practically Achievable? International journal of organ transplantation medicine, 2015. 6(3): p. 93-98.

4. Tullis, G.E., K. Spears, and M.D. Kirk, Immunological barriers to stem cell therapy in the central nervous system. Stem Cells Int, 2014. 2014: p. 507905.

5. Wood, K.J., et al., 81 - Concepts and Challenges in Organ Transplantation: Rejection, Immunosuppression, and Tolerance, in Clinical Immunology (Fifth Edition), R.R. Rich, et al., Editors. 2019, Content Repository Only!: London. p. 1097-1114.e1.

6. Mandal, P.K., et al., Efficient ablation of genes in human hematopoietic stem and effector cells using CRISPR/Cas9. Cell Stem Cell, 2014. 15(5): p. 643-52.

7. Torikai, H., et al., Genetic editing of HLA expression in hematopoietic stem cells to broaden their human application. Scientific reports, 2016. 6(1): p. 1-11.

8. Riolobos, L., et al., HLA engineering of human pluripotent stem cells. Mol Ther, 2013. 21(6): p. 123241.

9. Haga, K., et al., Permanent, lowered HLA class I expression using lentivirus vectors with shRNA constructs: Averting cytotoxicity by alloreactive T lymphocytes. Transplantation proceedings, 2006. 38(10): p. 3184-3188.

10. Cui, D., et al., Generating hESCs with reduced immunogenicity by disrupting TAP1 or TAPBP. Biosci Biotechnol Biochem, 2016. 80(8): p. 1484-91.

11. Wang, D., et al., Targeted Disruption of the B2-Microglobulin Gene Minimizes the Immunogenicity of Human Embryonic Stem Cells. Stem cells translational medicine, 2015. 4(10): p. 1234-1245.

12. Torikai, H., et al., ZFN-Driven Gene Editing Prevents HLA-A Expression On Hematopoietic Stem Cells Improving The Chance Of Finding An HLA-Matched Donor. Blood, 2013. 122(21): p. 1655-1655.

13. McLellan, M.A., N.A. Rosenthal, and A.R. Pinto, Cre-loxP-Mediated Recombination: General Principles and Experimental Considerations. Curr Protoc Mouse Biol, 2017. 7(1): p. 1-12.

14. Zimmer, A.M., et al., Loss-of-function approaches in comparative physiology: is there a future for knockdown experiments in the era of genome editing? J Exp Biol, 2019. 222(Pt 7).

15. Ran, F.A., et al., Genome engineering using the CRISPR-Cas9 system. Nature Protocols, 2013. 8(11): p. 2281-2308.

16. Torikai, $\mathrm{H}$. , et al., Toward eliminating HLA class I expression to generate universal cells from allogeneic donors. Blood, 2013. 122(8): p. 1341-1349.

17. 17. Qian, X., L.G. Villa-Diaz, and P.H. Krebsbach, Advances in culture and manipulation of human pluripotent stem cells. Journal of dental research, 2013. 92(11): p. 956-962. 
18. Chen, X., et al., Dual sgRNA-directed gene knockout using CRISPR/Cas9 technology in Caenorhabditis elegans. Scientific reports, 2014. 4: p. 7581.

19. Beerman, I., et al., Quiescent hematopoietic stem cells accumulate DNA damage during aging that is repaired upon entry into cell cycle. Cell Stem Cell, 2014. 15(1): p. 37-50.

20. Bauer, D.E., et al., An erythroid enhancer of BCL11A subject to genetic variation determines fetal hemoglobin level. Science, 2013. 342(6155): p. 253-7.

21. Sentmanat, M.F., et al., A Survey of Validation Strategies for CRISPR-Cas9 Editing. Scientific Reports, 2018. 8(1): p. 888.

22. 22. Hong, C.H., et al., Antigen Presentation by Individually Transferred HLA Class I Genes in HLA-A, HLA-B, HLA-C Null Human Cell Line Generated Using the Multiplex CRISPR-Cas9 System. J Immunother, 2017. 40(6): p. 201-210.

23. Xu, H., et al., Targeted Disruption of HLA Genes via CRISPR-Cas9 Generates iPSCs with Enhanced Immune Compatibility. Cell Stem Cell, 2019. 24(4): p. 566-578.e7.

24. Jang, Y., et al., Development of immunocompatible pluripotent stem cells via CRISPR-based human leukocyte antigen engineering. Experimental \& Molecular Medicine, 2019. 51(1): p. 1-11.

25. Mattapally, S., et al., Human Leukocyte Antigen Class I and II Knockout Human Induced Pluripotent Stem Cell-Derived Cells: Universal Donor for Cell Therapy. Journal of the American Heart Association, 2018. 7(23): p. e010239-e010239.

26. Hicklin, D.J., et al., beta2-Microglobulin mutations, HLA class I antigen loss, and tumor progression in melanoma. The Journal of clinical investigation, 1998. 101(12): p. 2720-2729.

\section{Tables}

Table 1.The guide RNAs targeting the B2M gene. The BME and BMI indicate the gRNAs targeting exon 1 and intron 1 respectively.

\begin{tabular}{|ll|}
\hline gRNA Name & gRNA Sequence \\
\hline BME & F: 5'- CACCGGGCCGAGATGTCTCGCTCCG- 3' \\
& R: 5'- AAACCGGAGCGAGACATCTCGGCCC- 3' \\
\hline BMI & F: 5'- CACCGAGCCCCATCAAGAGGTGGAT- 3' \\
& R: 5'- AAACATCCACCTCTTGATGGGGCTC- 3' \\
\hline
\end{tabular}




\begin{tabular}{|c|c|c|}
\hline Product length & Primer Sequence & Primer Name \\
\hline \multirow[t]{2}{*}{ 141bp } & F: 5'- TTCTTGGGTAGTTTGCAGTTTTAA- 3' & CrB2M_1 \\
\hline & R: 3'- CCCGGCTCTACAGAGCGAGGCCAAA- 5' & \\
\hline \multirow[t]{2}{*}{ 141bp } & F: 5'- TTCTTGGGTAGTTTGCAGTTTTAA- 3' & CrB2M_2 \\
\hline & R: 3'- CTCGGGGTAGTTCTCCACCTACAAA- 5' & \\
\hline \multirow[t]{2}{*}{ 2765bp } & F: 5'-CCTTGTCCTGATTGGCTGGG-3' & CrB2M_3 \\
\hline & R: 3'-TTTGCGTTTAGGGTGACAGTGTAC-5' & \\
\hline
\end{tabular}

Table2. The primer sequences used in this study.The CrB2M_1 and CrB2M_2 sequences were used to confirm proper integration of the gRNAs into the vector, each targeting exon 1 and intron 1 respectively. The CrB2M_3 served as the primer for genotyping the deleted.

\section{Figures}
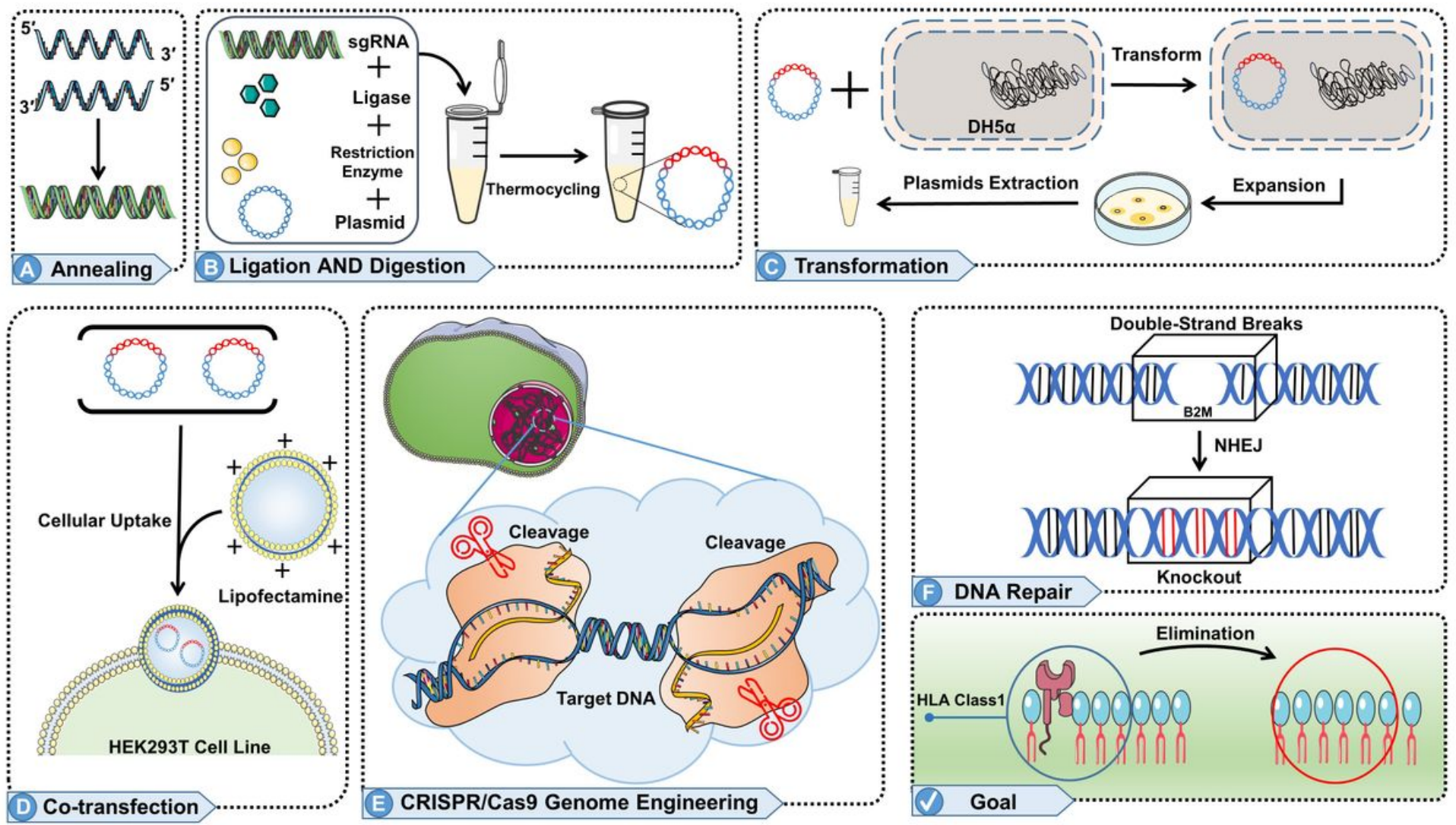

Figure 1

A Schematic flowchart of the experimental procedure for knocking out B2M gene out by CRISPR/Cas9 genome editing system.(1) Generating the desired sgRNA through designed guide oligos under temperature condition. (2) Inserting the sgRNA into backbone of PX458 vector (red region) followed by 
ejection of two-cutting selective sites. (3) Transforming the resulting vectors into E. coli DH5a and isolating vectors from colonies. (4) Co-transfecting the collected vectors into HEK293T cell line using commercially transfection reagent, Lipofectamine. (5) Directing the sgRNA into the specific genomic site and editing the targeted DNA trough Cas9 protein. (6) Rejoining the fragment sites by error-prone NHEJ pathway and knocking out B2M gene.
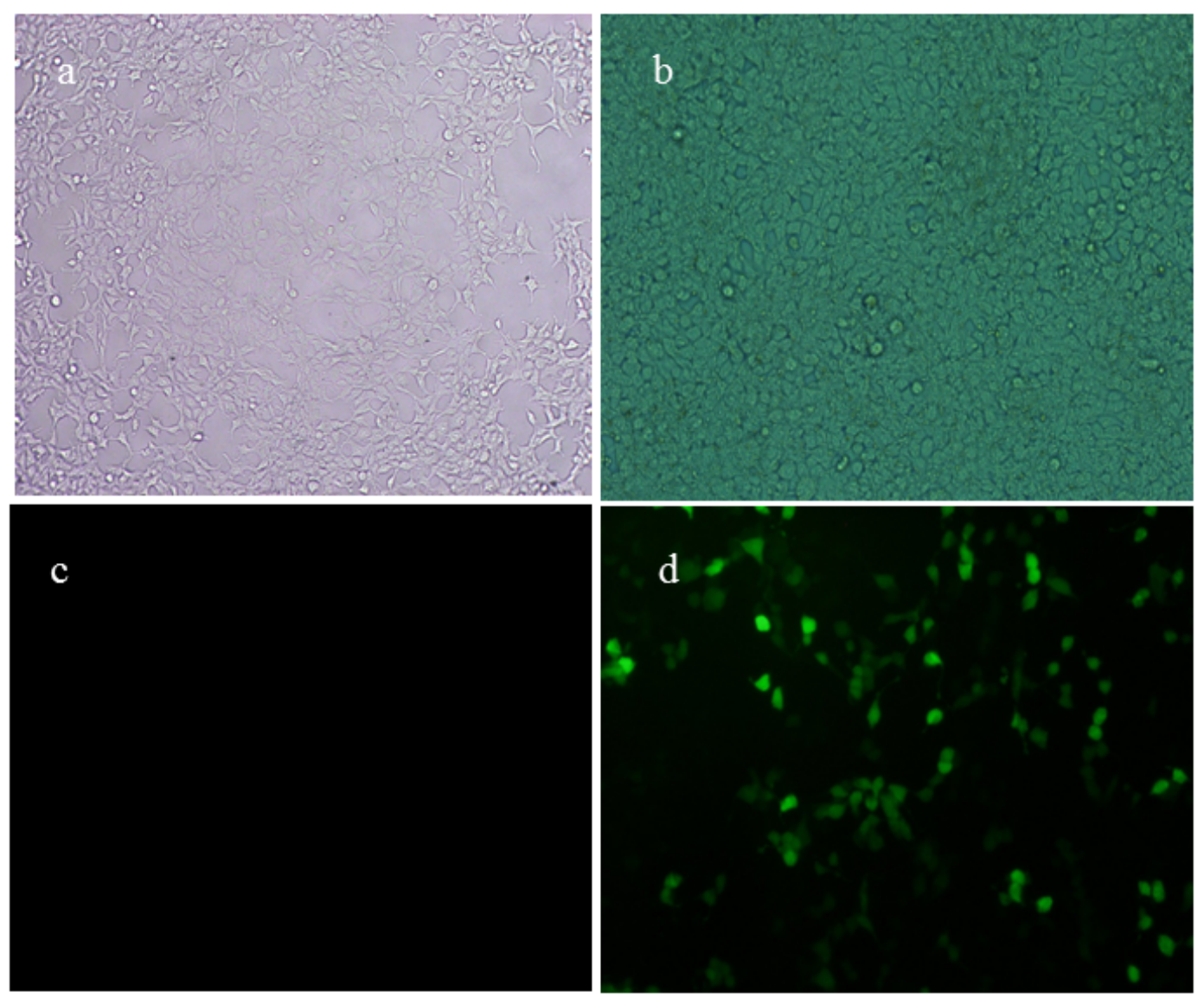

\section{Figure 2}

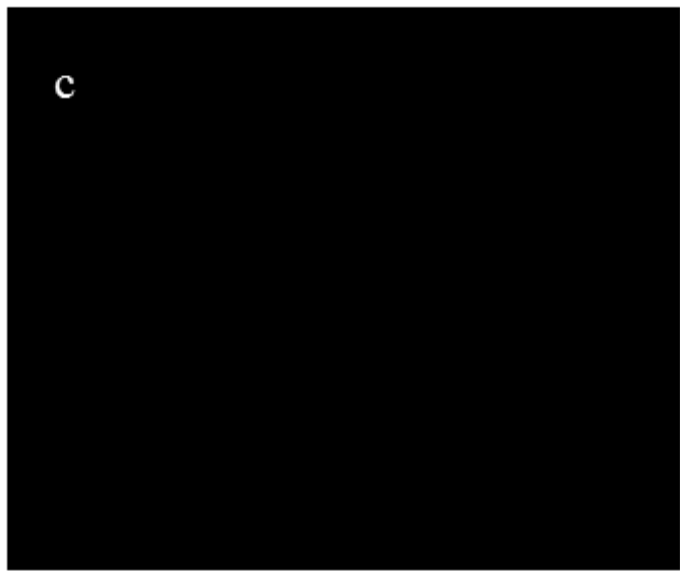

The GFP protein expression of HEK293 cells following transfection. The transfection was carried out using Lipofectamin reagents. The above figure illustrates a small proportion of the total cells, absorbing the vectors containing the gRNAs. (a) Untransfected HEK293T cells with UV visible. (b) Transfected HEK293T cells with UV visible. (c) Untransfected HEK293T cells with GFP-. (d) Transfected HEK293T cells with GFP+.

\section{Supplementary Files}

This is a list of supplementary files associated with this preprint. Click to download. 
- Manuscripttitlepage.docx

- coidisclosure.Dara.docx

- coidisclosure.Farid.docx

- coidisclosure.marjan.docx

- coidisclosure.maryamranjbar.docx

- coidisclosure.dianatpour.docx

- coverletter11.doc 